$\$=-1$

\title{
Initial Probability Distribution in Markov Chain Model for Fatigue Crack Growth Problem
}

\author{
Siti SarahJanuri ${ }^{1}$, Zulkifli Mohd Nopiah ${ }^{2}$, Ahmad kamal Ariffin Mohd Ihsan ${ }^{3}$, Nurulkamal Masseran ${ }^{4}$ and \\ Shahrum Abdullah ${ }^{5}$ \\ Faculty of Computer and Mathematical Sciences, Universiti Teknologi MARA Cawangan Negeri Sembilan, Kampus Seremban 70300 \\ Seremban, Negeri Sembilan, Malaysia \\ Faculty of Engineering and Built Environment, Universiti Kebangsaan Malaysia, 39000 UKM Bangi, Selangor, Malaysia \\ Faculty of Science and Technology, Universiti Kebangsaan Malaysia, 39000 UKM Bangi, Selangor, Malaysia \\ Corresponding Author Email: sitisarah@tmsk.uitm.edu.my
}

\begin{abstract}
Stochastic processes in fatigue crack growth problem usually due to the uncertainties factors such as material properties, environmental conditions and geometry of the component. These random factors give an appropriate framework for modelling and predicting a lifetime of the structure. In this paper, an approach of calculating the initial probability distribution is introduced based on the statistical distribution of initial crack length. The fatigue crack growth is modelled and the probability distribution of the damage state is predicted by a Markov Chain model associated with a classical deterministic crack Paris law. It has been used in calculating the transition probabilities matrix to represent the physical meaning of fatigue crack growth problem. The initial distribution has been determined as lognormal distribution which $66 \%$ that the initial crack length will happen in the first state. The data from the experimental work under constant amplitude loading has been analyzed using the Markov Chain model. The results show that transition probability matrix affect the result of the probability distribution and the main advantage of the Markov Chain is once all the parameters are determined, the probability distribution can be generated at any time, $x$.
\end{abstract}

Keywords: Initial distribution, Markov Chain model, Paris law equation, fatigue crack growth

\section{Introduction}

Probability and statistics have been applied in the fatigue crack growth problem analysis since decades. It proved that fatigue crack growth data contains statistical nature, and therefore the data and analysis be applied statistically. It was observed that fatigue crack growth also contains certain statistical nature, and therefore would better be treated statistically [15] . Fatigue of a structural material is the most common cause of damage in engineering such as machine components. A fatigue phenomenon is a complexity process and thus, the modelling is difficult. The deep and depth analysis regarding fatigue crack growth is required to guarantee the result is reliable. Fatigue crack growth process is an integrated of random factors such as inhomogeneity of real material, manufacturing processes, number of loading, geometry of component, condition of technological and environmental condition [5][2]. These random factors explained the influencing of the uncertainty factors to the fatigue crack growth process and it contributes to the scattering of the crack size.

There is a scattering of statistical fatigue test results, and it is considering many random factors to describe the whole process of fatigue crack growth. Therefore, probabilistic method has brought great concerned to the research area to replace deterministic method in determining fatigue life prediction [9] [11]. Probabilistic also known as stochastic with two types of the crack growth model: randomization of the deterministic model with fatigue crack growth model by providing the distribution of random time and secondly, randomization of the Paris-Erdogan equation [5] , [14] . In addition, probabilistic method is interested to study because of the dependant of the microstructure [12].

There are still many more research studies that have used the Markov chain approach to evaluate and model the stochastic behaviors for the data of fatigue crack growth. Unfortunately, studies of the initial distribution have not been common. This study proposes probability distribution of initial crack length to explain the initial probability distribution and deterministic Paris law equation to explain the fatigue crack growth process. The aim of this paper is to present the procedure of determination of the probability distribution of material Aluminium Alloy 7075 in the cooperation with Markov Chain model.

\section{Materials and Method}

\subsection{Markov Chain Model for the Fatigue Crack Growth Problem}

Markov Chain model is a proper tool that considered complexity or size of the system in the modeling [13]. System dependability of Markov Chain are widely measured by performance, availability, reliability and safety [4], and various Markov models have been solved by different solution techniques [9] .

The Markov Chain model is defined as a technique for modelling the system state transitions and calculating the probability of reaching various system states. Determining initial probability 
distribution is important to determine the future prediction. Previous study by [3] used empirical study of weld to calculate the lifetime of the Virkler's data, 2024-T3 aluminium alloy CCT specimens. Then, [1] believed that all the initial crack length should be started in the first state, i.e. the initial probability distribution is $p_{0}=[1,0,0, \ldots, 0]$.

There are few methods have been used to compute the transition probability matrix. Initially, [3] used Paris law equation, then, followed by [8] applied modified Paris law equation and [1] solved calculations of system of equation of mean and variance of load cycle to determine the probability values. Another study by [10] used a negative binomial distribution for calculating the transition probability functions of the pit depths.

A duty cycle (DC) is a repetitive period of operation in the component lifetime during which damage can accumulate. It is assumed that the increment of damage at the end of a DC depends on the probabilistic factor which the damage accumulation at the beginning of a DC and on the DC itself. The damage happens in at the beginning of DC is independent. How damage accumulates within a DC is not a matter of concern. Number of DC's is measured by the time, $\mathrm{x}=0,1,2, \ldots$ This assumption familiar with the Markov assumption (Kozin \& Bogdanoff, 1981).

\subsection{Methodology}

Markov Chain model is defined as a mathematical model for predicting the future state that depends only on the current state. For a given system, the Markov model consists of the number of possible states, the possible transition paths between these states, and the probability values of these transitions. Figure 1 depicts the transition probabilities change from the state to the next state as known as Markov Chain model with a circle is representing a state and arrows denoting the transition path between states.

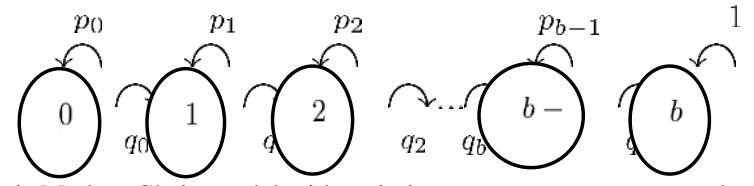

Figure-1. Markov Chain model with a circle represents a state. $p_{i}$ and $q_{i}$ are probabilities in particular state and changing state, respectively [3].

In Markov model, a duty cycle (DC) is an important element to introduce as a time to reach crack growth or as the group of the number of cycles accumulated to make a transition to next crack length [7]. The growth process of fatigue crack length was defined as a discrete both in time and states stochastic process. Markov property has two important elements: determining the initial distribution and the transition probability matrix. This study proposes that initial probability distribution is using Lognormal probability distribution which as the best distribution to describe the initial crack length distribution. The probability distribution is as follows:

$f(x ; \mu, \sigma)=\frac{1}{x \sigma \sqrt{2 \pi}} \exp \left[\frac{-(\ln (x)-\mu)^{2}}{2 \sigma^{2}}\right], \mu \geq 0, \sigma \geq 0$

Where, $\mu$ is a shape parameter and $\sigma$ is a scale parameter of the length of the initial crack. Lognormal distribution was chosen as the best distribution through the comparison of Kolmogorov Smirnov value from all three distributions (Lognormal, normal and Weibull). While, the transition probability matrix values were computed to show the process of fatigue crack growth happened. Classical Paris law equation was applied to compute the probability values.

The whole procedures of estimating the probability distribution of the states are as in following steps. Initially, defining the time, $x=0,1,2, \ldots$ is measured by the number of duty cycles. Then, number of damage states, $s=1,2, \ldots, b$ where the state $b$ denotes a failure state. The duty cycle is specified in a $b \times b$ probability transition matrix, $P$ as

$$
P=\left(\begin{array}{ccccccc}
p_{0} & q_{0} & 0 & 0 & \cdots & 0 & 0 \\
0 & p_{1} & q_{1} & 0 & \cdots & 0 & 0 \\
0 & 0 & p_{2} & q_{2} & \cdots & 0 & 0 \\
\vdots & \vdots & \vdots & \vdots & \cdots & \vdots & \vdots \\
0 & 0 & 0 & 0 & \cdots & p_{b-1} & q_{b-1} \\
0 & 0 & 0 & 0 & \cdots & 0 & 1
\end{array}\right)
$$

Where

$p_{j} \geq 0, \quad p_{j}+q_{j}=1$

$p_{j}=$ Probability $\{$ remain in state $\mathrm{j} \mid$ initially in state $\mathrm{j}\}$

$q_{j}=$ Probability $\{$ go to state $\mathrm{j}+1 \mid$ initially in state $\mathrm{j}\}$

Next, the number of states was determined which is crack length increment as a state. The states are assumed to be discrete with the states, $s=1,2, \ldots, b$, where $b$ corresponds to failure state. In the Markov chain model, it is assumed that crack length $\delta a$ increases by stage. The calculation of each state as in Equation (2).

$a_{j}=a_{0}+j \delta a, j=0,1,2, \ldots, b$

where, $a_{j}$ is crack length in state $j(m m)$, and $a_{0}$ is initial crack length $(\mathrm{mm})$.

Then, estimating the initial probability $(1 \times b)$ row vector by using distribution of initial crack length was using lognormal distribution. The probability of being in state $j$ at the time $x$ is given Equation (3).

$p_{0}=\left\{\pi_{0}, \pi_{1}, \pi_{2}, \ldots, \pi_{b-1}, \pi_{b}\right\}$

where $\pi_{j}=$ Probability $\{$ damage is in state $\mathrm{j}$ at time $\mathrm{x}=0\}$ and $\sum_{1}^{b-1} \pi_{j}=1, \pi_{b}=0$.

The assumption that, $\pi_{b}=0$ means that no component is in the failed state $b$ in initial state.

Then, the transition probabilities matrix $(b \times b)$ is computed. In this study, transient probability $q_{j}$ can be obtained by applying a Paris law equation into the model to represent the physical meaning of fatigue damage. The probability of changing to next state, $q_{j}$ can be defined as stress intensity factor function as in Equation (4) which is the equation was derived from Equation (5).

increment, $C, m=$ material constant, and $\Delta K=$ stress intensity factor range $[M P a \sqrt{m}$. Then, by solving system differential equation, $q=C / \delta a(\Delta K)^{m}$

$\Delta K=2 / \pi(\Delta \sigma \sqrt{\pi} a)$ where

$\Delta \sigma=$ stress difference.

Lastly, Markov property value is computed by using Equation (6).

$q_{j}=q\left(\Delta K_{j}\right)$

Paris law equation: $d a / d N=C(\Delta K)^{m}$

where $d a=$ crack length increment $(\mathrm{mm}), d N=$ number of cycles $p_{x}=p_{0} P^{x}$

Equation (6) explains the probability is changing due to time changes. A comparison can be made for several times in order to know the probability in the future in any time (duty cycle), $x$. 
This study focuses on the performance of the Markov Chain model to estimate the probability distribution of damage state. In this paper, transition probability matrix was calculated using the classical Paris law equation. Whilst, the initial probability distribution of the initial state was computed based on Lognormal probability distribution which is more accurately in describing the distribution of the initial crack length.

\subsection{Fatigue Data}

Fatigue crack growth data are required to explain the applicability of the probabilistic models. The methodology was demonstrated using the experimental observations from a material of Aluminium alloy A7075-T6. Ten samples were tested in the experiment. The dimensions of the specimens are $160.0 \mathrm{~mm}$ wide and $60.0 \mathrm{~mm}$ thick. The experiment was tested at room temperature under constant amplitude loading of $45 \mathrm{kN}$ stress load and 0.1 fixed stress ratios in order to observe the fatigue crack growth on the surface of the material. The use of $45 \mathrm{kN}$ ensures that it exceeded the endurance limit load which is to affirm that fatigue occurs for the dimension of this material. The length of the crack growth of the ten specimens was measured using the digital calliper and the length taken is the average of five times measurement. The results from the experiment as in Figure 2 shows there were five stages of fatigue crack growth starting from initial crack length and end with failure part.

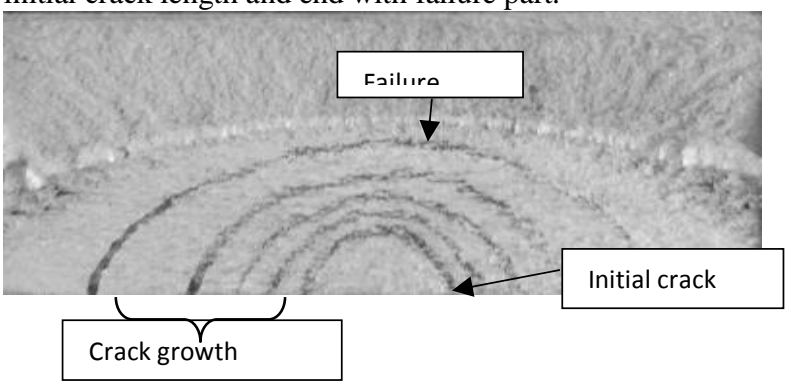

Figure-2: Material surface of the aluminium alloy 7075-T6 from the experiment

Figure 3 depicts the crack lengths $(a)$ against the number of cycles $(N)$ for ten specimens from the experiments. From Paris law equation, $\Delta K$ was evaluated using $\Delta K=2 / \pi(\Delta \sigma \sqrt{\pi a})$. It shows scattering for the initial crack lengths from specimen to specimen variability. Therefore, the probability distribution for the initial crack was determined to represent the scatter of the data.

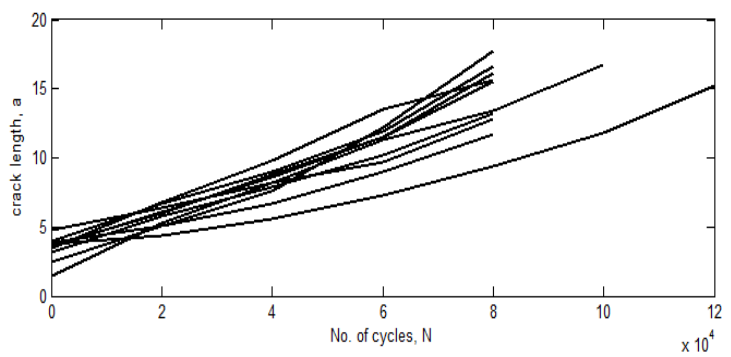

Figure-3. Crack length $(a)$ versus number of cycles $(N)^{\mathrm{x}}$

\section{Results and Discussion}

In this paper, probability distribution for damage state was demonstrated by using Markov property. Markov Chain model was constructed with duty cycles are 2000 cycles and $\delta a=0.3 \mathrm{~mm}$. Probability distribution of initial state and transition probability matrix were discussed as follows. Table 1 explains the probability values of initial state, $p_{0}$ which was derived from the lognormal distribution. States are defined as the crack length increment. It starts with initial crack length part and ends with failure part. The highest probability value of initial crack length implies logically at the first and second state, that is $66 \%$ and $33 \%$, respectively. Then, the values are decreasing gradually through the states which all the values are approximately to $0 \%$. Therefore, it can be said that the initial crack length is impossible to be happened in other states except at the first and second state.

Table-1. Initial probability values for each state

\begin{tabular}{|c|c|c|c|c|c|c|c|c|c|}
\hline State, $j$ & $p_{0}$ & State, $j$ & $p_{0}$ & State, $j$ & $p_{0}$ & State, $j$ & $p_{0}$ & State, $j$ & $p_{0}$ \\
\hline 0 & 0.66 & 10 & $\approx 0$ & 20 & $\approx 0$ & 30 & $\approx 0$ & 40 & 0 \\
\hline 1 & 0.33 & 11 & $\approx 0$ & 21 & $\approx 0$ & 31 & $\approx 0$ & 41 & 0 \\
\hline 2 & 0.001 & 12 & $\approx 0$ & 22 & $\approx 0$ & 32 & $\approx 0$ & 42 & 0 \\
\hline & $\approx 0$ & 13 & $\approx 0$ & 23 & $\approx 0$ & 33 & $\approx 0$ & 43 & 0 \\
\hline 4 & $\approx 0$ & 14 & $\approx 0$ & 24 & $\approx 0$ & 34 & $\approx 0$ & 44 & 0 \\
\hline 5 & $\approx 0$ & 15 & $\approx 0$ & 25 & $\approx 0$ & 35 & $\approx 0$ & 45 & 0 \\
\hline 6 & $\approx 0$ & 16 & $\approx 0$ & 26 & $\approx 0$ & 36 & $\approx 0$ & 46 & 0 \\
\hline 7 & $\approx 0$ & 17 & $\approx 0$ & 27 & $\approx 0$ & 37 & $\approx 0$ & 47 & 0 \\
\hline 8 & $\approx 0$ & 18 & $\approx 0$ & 28 & $\approx 0$ & 38 & $\approx 0$ & 48 & 0 \\
\hline 9 & $\approx 0$ & 19 & $\approx 0$ & 29 & $\approx 0$ & 39 & 0 & 49 & 0 \\
\hline
\end{tabular}

Then, the transition probabilities matrix was calculated from the classical Paris law equation. The resulting matrix $\mathrm{P}$ was used to determine the probability distributions for interesting fatigue crack illustration of the Markov Chain model, a simple example is given. It is assumed that number of damage states is 50 and the lengths. As an

Table-2. E.g. Probability distribution of the state

\begin{tabular}{|c|c|c|c|c|c|c|c|c|c|}
\hline State, $j$ & $p_{0}$ & State, $j$ & $p_{0}$ & State, $j$ & $p_{0}$ & State, $j$ & $p_{0}$ & State, $j$ & $p_{0}$ \\
\hline 0 & 0.017696 & 10 & $\approx 0$ & 20 & $\approx 0$ & 30 & $\approx 0$ & 40 & 0 \\
\hline 1 & 0.086671 & 11 & $\approx 0$ & 21 & $\approx 0$ & 31 & $\approx 0$ & 41 & 0 \\
\hline 2 & 0.192718 & 12 & $\approx 0$ & 22 & $\approx 0$ & 32 & $\approx 0$ & 42 & 0 \\
\hline 3 & 0.256843 & 13 & $\approx 0$ & 23 & $\approx 0$ & 33 & $\approx 0$ & 43 & 0 \\
\hline 4 & 0.2280 & 14 & $\approx 0$ & 24 & $\approx 0$ & 34 & $\approx 0$ & 44 & 0 \\
\hline 5 & 0.141579 & 15 & $\approx 0$ & 25 & $\approx 0$ & 35 & $\approx 0$ & 45 & 0 \\
\hline 7 & 0.019878 & 17 & $\approx 0$ & 27 & $\approx 0$ & 37 & $\approx 0$ & 47 & 0 \\
\hline 8 & 0.00441 & 18 & $\approx 0$ & 28 & $\approx 0$ & 38 & $\approx 0$ & 48 & 0 \\
\hline
\end{tabular}


Lastly, probability distribution was computed using Equation 6. A comparison between the probability distributions of the state damage is possible because the initial state vector is the same. It indicates that the probability of being in a state, e.g. state 2, after 10 duty cycles depends greatly on the transition probability. Thus, the probability of being in state 2 is changing from the initial value 0.001 to 0.1927 , respectively. The advantages of the Markov Chain the model is capable and easy to calculate the probability of state of damage at any time, $x$, using Markov property when all the parameter values are determined.

\section{Conclusion}

Probability distribution of the damage state of the material aluminium alloy, A7075-T6 is analysed in this study by using Markov Chain model. The factors that contributing to the fatigue are the probability distribution of initial crack length and the stress intensity factor that includes the width and the length on the specimen, the number of loading cycles and the stress ratio. The probability distribution of the initial crack length is important to determine probability distribution for interesting fatigue crack length due to the uncertainty factors for this variable. This study only focuses on the deterministic factor for calculating the transition probability matrix. It is suggested to include the random factors in the model to calculating the transition probability matrix and sees the performance of the model for capturing fatigue life prediction. The advantage of the Markov Chain model is the capability of the model in capturing the results. Once model parameters are determined, the state of damage in the given structure is available at any time using Markov property. This means that all statistical information about the damage process can be represented by the model.

\section{References}

[1] Drewniak, J., \& Hojdys, L. (2015). The Method of Analysis of Fatigue Crack Growth by Bogdanow-Kozin Model. Machine Dynamics Research, 39(4), 125-132.

[2] Ellyin, F. (1997). Fatigue Damage, Crack Growth and Life Prediction. Chapman \& Hall, 2-6 Boundary Row, London SE18HN, UK.

[3] Gansted, L., Brincker, R., \& Hansen, L. P. (1991). Fracture mechanical Markov chain crack growth model. Engineering Fracture Mechanics, 38(6), 475-489. http://doi.org/10.1016/0013-7944(91)90097-K

[4] Khelif, R., Chateauneuf, A., \& Chaoui, K. (2008). Statistical analysis of HDPE fatigue lifetime. Meccanica, 43(April), 567576. http://doi.org/10.1007/s11012-008-9133-7

[5] Kocańda, D., \& Jasztal, M. (2012). Probabilistic predicting the fatigue crack growth under variable amplitude loading. International Journal of Fatigue, 39, 68-74. http://doi.org/10.1016/j.ijfatigue.2011.03.011

[6] Kozin, F., \& Bogdanoff, J. (1981). A Critical Analysis of Some Probabilistic Models for Fatigue Crack Growth. Engineering Fracture Mechanics, 14(MC), 59-89.

[7] Kozin, F., \& Bogdanoff, J. L. (1983). On the probabilistic modeling of fatigue crack growth. Engineering Fracture Mechanics, 18(3), 623-632. http://doi.org/10.1016/00137944(83)90055-3

[8] Lee, O. S. (1998). Fatigue Life Prediction by Statistical Approach Under Constant Amplitude Loading. KSME International Journal, 12(1).

[9] Makkonen, M. (2009). Predicting the total fatigue life in metals. International Journal of Fatigue, 31(7), 1163-1175. http://doi.org/10.1016/j.ijfatigue.2008.12.008

[10] Ossai, C. I., Boswell, B., \& Davies, I. (2016). Markov chain modelling for time evolution of internal pitting corrosion distribution of oil and gas pipelines. Engineering Failure Analysis, 60, 209-228 http://doi.org/10.1016/j.engfailanal.2015.11.052

[11] Rastogi, R., Ghosh, S., Ghosh, A. K., Vaze, K. K., \& Singh, P. K. (2016). Fatigue crack growth prediction in nuclear piping using Markov chain Monte Carlo simulation. Fatigue \& Fracture of Engineering Materials \& Structures. http://doi.org/10.1111/ffe.12486

[12] Santecchia, E., Hamouda, A. M. S., Musharavati, F., Zalnezhad, E., Cabibbo, M., Mehtedi, M. El, \& Spigarelli, S. (2016). A Review on Fatigue Life Prediction Methods for Metals. Advances in Materials Science and Engineering Volume, 2016. http://doi.org/10.1155/2016/9573524

[13] Son, K. S., Kim, D. H., Kim, C. H., \& Kang, H. G. (2016). Study on the systematic approach of Markov modeling for dependability analysis of complex fault-tolerant features with voting logics. Reliability Engineering \& System Safety, 150, 44-57. http://doi.org/10.1016/j.ress.2016.01.014

[14] Wu, W. F., \& Ni, C. C. (2003). A study of stochastic fatigue crack growth modeling through experimental data. Probabilistic Engineering Mechanics, 18(2), 107-118. http://doi.org/10.1016/S0266-8920(02)00053-X

[15] Wu, W. F., \& Ni, C. C. (2004). Probabilistic models of fatigue crack propagation and their experimental verification. Probabilistic Engineering Mechanics, 19, 247-257. http://doi.org/10.1016/j.probengmech.2004.02.008 